Original Article

\title{
EFFECTS OF FORMULATION PARAMETERS ON PROPERTIES OF GASTRIC FLOATING TABLETS CONTAINING POORLY SOLUBLE DRUG: DICLOFENAC SODIUM
}

\author{
SITHA KIMa, EKAPOL LIMPONGSA ${ }^{b, c^{*}}$, NAPAPHAK JAIPAKDEEb,c \\ aGraduate School, Khon Kaen University, Khon Kaen, 40002, Thailand, bDivision of Pharmaceutical Technology, Faculty of Pharmaceutical \\ Sciences, Khon Kaen University, Khon Kaen, 40002, Thailand, cCenter for Research and Development of Herbal Health Products, Khon \\ Kaen University, Khon Kaen, 40002, Thailand \\ Email: ekapol@hotmail.com
}

Received: 03 Aug 2018, Revised and Accepted: 04 Sep 2018

\begin{abstract}
Objective: The objective of this study was to prepare and investigate the effects of formulation parameters on the properties of gastric floating tablets containing diclofenac sodium (DICL) as a model of poorly soluble acidic drug, sodium bicarbonate $\left(\mathrm{NaHCO}_{3}\right)$ or calcium carbonate $\left(\mathrm{CaCO}_{3}\right)$ as gas-forming agent, hydroxypropyl methylcellulose (HPMC) K100M or K15M as swelling polymer and sodium lauryl sulfate (SLS) as wetting agent.
\end{abstract}

Methods: DICL floating tablets were prepared using direct compression method. The compressed tablets were evaluated for tablet properties, swelling index, and in vitro buoyancy. The in vitro release under non-sink condition was determined. Molecular interaction was studied using differential scanning calorimetry and fourier transform infrared spectroscopy.

Results: The tablet properties of all DICL floating tablets were within the acceptance criteria. The molecular interaction between DICL and excipients in the formulation was excluded. Depends on the formulation compositions, the swelling index at $3 \mathrm{~h}$ (SI) ranged from $44 \pm 11$ to $1158 \pm 33$ $\%$, whereas the buoyancy properties namely floating lag time (FLT) and total floating time (TFT) were $0.33 \pm 0.03$ to $10.04 \pm 0.04 \mathrm{~min}$ and $10.0 \pm 0.0$ to $>12 \mathrm{~h}$, respectively. $\mathrm{NaHCO}_{3}$ showed higher swelling, buoyancy and release properties compared to those of $\mathrm{CaCO}_{3}$. $\mathrm{NaHCO}_{3}$ at $20 \%$ gave sufficient swelling (SI of $1074 \pm 16 \%$ ), buoyancy (FLT of $0.39 \pm 0.03 \mathrm{~min}$, TFT of $>12 \mathrm{~h}$ ) and release properties (cumulative release of $5.83 \pm 0.02 \%$ ). HPMC K100M showed better swelling property of which its initial swelling rate was $1412 \pm 25 \% / \mathrm{h}$ compared to HPMC K15M (1042 $\pm 31 \% / \mathrm{h})$. HPMC K100M at 20\% showed better buoyancy and release properties compared to those obtained from HPMC K100M at 30\%. The release testing under non-sink conditions was able to distinguish the effect of formulation parameters on the DICL release profiles. Incorporation of SLS at $0.25 \%$ could enhance both release rate and cumulative release of DICL from the floating tablets. Nevertheless, it showed the unacceptable adverse effect on swelling and buoyancy properties of DICL floating tablets. The TFT of DICL floating tablets containing $0.25 \%$ SLS was only $0.5 \pm 0.0 \mathrm{~h}$.

Conclusion: DICL floating tablets were successfully prepared. Tablets possessing suitable swelling and buoyancy properties were obtained using $\mathrm{NaHCO}_{3}$ at $20 \%$ as a gas-forming agent, with HPMC K100M at 10 and $20 \%$ as floating matrix and swelling polymer. Addition of SLS as wetting and solubilizing agent showed the unacceptable adverse effect on the swelling and buoyancy properties of DICL floating tablets. The release under sink conditions and/or in vivo pharmacokinetic studies shall be further performed.

Keywords: Diclofenac sodium, Floating tablet, HPMC, SLS

(C) 2018 The Authors. Published by Innovare Academic Sciences Pvt Ltd. This is an open-access article under the CC BY license (http://creativecommons.org/licenses/by/4.0/) DOI: http://dx.doi.org/10.22159/ijap.2018v10i6.28935

\section{INTRODUCTION}

Oral administration is the most preferred route among all other routes of administration used for systemic action. This route is widely used due to its ease of administration, and thus patient compliance can be improved [1]. However, the absorption of orally administered drugs has been impacted by several factors, including physicochemical properties of the drugs, gastric resident time (GRT) and localization of the drugs in the gastrointestinal tract.

Floating drug delivery systems (FDDSs) were developed to extend the GRT of the dosage form in order to improve the bioavailability of drugs [2]. Such systems are characterized by the buoyancy of the dosage form to float over the gastric content for an extended period of time due to their relatively low density, compared to the density of gastric fluid [3,4]. While the drug is floating over the gastric content, it is slowly released at the desired controlled-rate, resulting in higher bioavailability, enhanced therapeutic efficacy, and shorter time intervals for drug administration, which in turn improves patient compliance [5]. FDDSs are of particular interest for drugs which: (a) act locally in the stomach; (b) are primarily absorbed in the stomach; (c) are poorly soluble at an alkaline $\mathrm{pH}$; (d) have a narrow window of absorption; and (e) are unstable in the intestinal or colonic environment [6]. Furthermore, most of the drugs developed using the FDDSs have a short half-life and are generally well soluble in acidic medium. FDDSs include effervescent systems, of which important compositions are gas-forming agents and swellable polymers [7]. The effects of these compositions have been studied [8-10]. Nevertheless, limit study has been performed with the FDDSs of poorly soluble in acidic drugs.

Diclofenac sodium (DICL) is a nonsteroidal anti-inflammatory drug used to relieve pain and inflammation. Generally, DICL needs to be administered several times a day in order to maintain its therapeutic effects as DICL has short half-life [11]. DICL is a moderate weakly acidic drug $\left(\mathrm{pK}_{\mathrm{a}} 4.0\right)$ [12]. Its solubility in an acidic $\mathrm{pH}$ environment is extremely low.

Various methods are reported to improve the release of poorly soluble drugs, including (a) reduction of particle size to increase surface area, and thus increasing the release rate of drug; (b) formation of water-soluble complexes; (c) drug derivatization; (d) manipulation of solid state of drug substance to improve drug release; and (e) solubilization in surfactant systems [13,14].

Sodium lauryl sulfate (SLS) is the most popular surfactant used in oral drug delivery $[15,16]$. It is an anionic surfactant used in the formulation of tablets as the solubilizing agent to increase the solubility of the poorly soluble drug, by enhancing hydrophilic environment and wettability in the tablets matrix [17]. Presently, there is very little information on the impact of SLS on the properties of floating tablets.

Therefore, the objective of this present work was to prepare and investigate the effect of formulation parameters on the properties of gastric floating tablets containing diclofenac sodium (DICL) as a model of poorly soluble acidic drug, sodium bicarbonate $\left(\mathrm{NaHCO}_{3}\right)$ 
or calcium carbonate $\left(\mathrm{CaCO}_{3}\right)$ as gas-forming agent, hydroxypropyl methylcellulose (HPMC) K100M or K15M as swelling polymer, and sodium lauryl sulfate (SLS) as wetting agent.

\section{MATERIALS AND METHODS}

\section{Materials}

Diclofenac sodium (DICL) was obtained as a gift sample from T. 0 . Chemicals (1979) Co., Ltd., Bangkok, Thailand. Hydroxypropyl methylcellulose (HPMC K100M and $\mathrm{K} 15 \mathrm{M}$ ) were provided by Maxway Pharmaceutical Co., Ltd., Bangkok. Microcrystalline cellulose (MCC; Avicel PH102) was bought from Onimax Co., Ltd., Bangkok, Thailand. Sodium bicarbonate $\left(\mathrm{NaHCO}_{3}\right)$, calcium carbonate $\left(\mathrm{CaCO}_{3}\right)$, sodium lauryl sulfate (SLS), magnesium stearate, and silicon dioxide (Aerosil) were received from K Science Center and Medical Co., Ltd., Khon Kaen, Thailand.

\section{Solubility study}

The solubility of DICL in $0.1 \mathrm{~N}$ hydrochloric $(\mathrm{HCl}), 0.1 \mathrm{~N} \mathrm{HCl}$ containing 1 and $2 \%$ SLS media was determined. The excessive amount of DICL was added into $10 \mathrm{ml}$ of each medium in test tube $(n=3)$. The mixture was sonicated for $30 \mathrm{~min}$ and then shaken at 80 rpm for $24 \mathrm{~h}$ using a shaking water bath (LSB-030S, Labtech, Korea) at a maintained temperature of $37 \pm 0.5{ }^{\circ} \mathrm{C}$. The saturated solution was filtrated through nylon syringe filter $(0.45 \mu \mathrm{m}, 13 \mathrm{~mm}$, Millipore filter, Millipore, Bedford, MA). The clear supernatant was appropriately diluted and determined using UV-Visible spectrophotometer (UV-1201, Shimadzu, Japan) at wavelength $276 \mathrm{~nm}$.

\section{Preparation of DICL floating tablets}

DICL floating tablets were prepared using the direct compression method. The drug, MCC, $\mathrm{HPMC}, \mathrm{NaHCO}_{3}$ or $\mathrm{CaCO}_{3}$ with or without SLS were accurately weighed and mixed in a blender for $10 \mathrm{~min}$. The blended powder was subsequently lubricated with magnesium stearate and Aerosil for $3 \mathrm{~min}$. The obtained drugexcipients mixture was compressed at the force of $60 \mathrm{kgf} / \mathrm{cm} 2$ using the hydrostatic press machine (Model 3126, Shimadzu, Japan) without holding time. The floating formulations of DICL tablets are shown in table 1.

Table 1: Compositions of DICL floating tablets (100 mg-DICL per tablet)

\begin{tabular}{|c|c|c|c|c|c|c|c|c|c|c|}
\hline \multirow{2}{*}{$\begin{array}{l}\text { Composition } \\
(\%)\end{array}$} & \multicolumn{10}{|c|}{ Formulation } \\
\hline & F1 & F2 & F3 & F4 & F5 & F6 & F7 & FS1 & FS2 & FS3 \\
\hline DICL & 40 & 40 & 40 & 40 & 40 & 40 & 40 & 40 & 40 & 40 \\
\hline HPMC K100M & 20 & 20 & 20 & 20 & 10 & 30 & - & 20 & 20 & 20 \\
\hline HPMC K15M & - & - & - & - & - & - & 20 & - & - & - \\
\hline $\mathrm{NaHCO}_{3}$ & - & 20 & 10 & 30 & 20 & 20 & 20 & 20 & 20 & 20 \\
\hline $\mathrm{CaCO}_{3}$ & 20 & - & - & - & - & - & - & - & - & - \\
\hline SLS & - & - & - & - & - & - & - & 0.25 & 0.5 & 1 \\
\hline Aerosil & 0.5 & 0.5 & 0.5 & 0.5 & 0.5 & 0.5 & 0.5 & 0.5 & 0.5 & 0.5 \\
\hline Mg stearate & 1 & 1 & 1 & 1 & 1 & 1 & 1 & 1 & 1 & 1 \\
\hline MCC & 18.5 & 18.5 & 28.5 & 8.5 & 28.5 & 8.5 & 18.5 & 18.25 & 18 & 17.5 \\
\hline
\end{tabular}

\section{Evaluation of DICL floating tablets}

\section{Weight variation}

The weight variation was determined through random selections of twenty DICL floating tablets from each batch. The selected tablets were then weighed individually and accurately using an electronic balance (GF-600, AND, Japan), and the average weight was determined.

\section{Thickness}

The thickness of the DICL floating tablets was measured using a vernier caliper (SM-112, TECLOCK, Japan). Ten tablets of each batch were randomly selected.

\section{Hardness}

The hardness test was conducted to measure the tablet strength. Ten tablets were selected randomly from each formulation for hardness evaluation using tablet hardness tester (VK200, Vankel, UK).

\section{Friability}

Friability evaluation was carried out using friability apparatus (Vankel, U. S.). 26 DICL floating tablets (6.5 g) were selected randomly from each batch. All tablets were weighted initially $\left(W_{\mathrm{i}}\right)$ and put into the friability apparatus. The tables were placed into the drum and rotated for 100 revolutions in $4 \mathrm{~min}$. Then, the tablets were removed, de-dusted and then weighted again $\left(W_{\mathrm{f}}\right)$. The percentage of friability was calculated using equation (1) [18].

$$
\% \text { Friability }=\frac{\left(W_{\mathrm{i}}-W_{\mathrm{f}}\right)}{W_{\mathrm{i}}} \times 100
$$

\section{Drug content}

Ten tablets of each DICL floating formulation were selected and individually weighed, and then triturated in a mortar. A quantity of powder equivalent to $100 \mathrm{mg}$ of drug was transferred into $100 \mathrm{ml}$ volumetric flask, and mixed up with methanol (HPLC grade) to the volume. The sample solution was mixed thoroughly and filtered using $0.45 \mu \mathrm{m}$ filter. The filtrate was diluted to the suitable concentration and analyzed for drug content at a wavelength of 276 $\mathrm{nm}$, using methanol as the blank by UV-Visible spectrophotometer (UV-1201, SHIMADZU, Japan) [6, 19].

\section{Swelling index}

Six tablets of each formulation were weighed $\left(W_{\mathrm{i}}\right)$ and placed into a separate beaker containing $100 \mathrm{ml}$ of $0.1 \mathrm{~N} \mathrm{HCl}$ solution, maintained at $37 \pm 0.5^{\circ} \mathrm{C}$ using circulation water bath (WiseCircu, Korea) [6]. At regular time intervals, the tablets were taken out of the medium and blotted with tissue paper to remove the excessive water on the surface of tablets. The swollen tablets were weighed again $\left(W_{\mathrm{f}}\right)$ and calculated for swelling index using equation (2)

$$
\text { Swelling index }=\frac{\left(W_{\mathrm{f}}-W_{\mathrm{i}}\right)}{W_{\mathrm{i}}} \times 100
$$

\section{In vitro buoyancy}

Buoyancy properties of DICL floating tablets were determined simultaneously with in vitro release study. The duration the tablets rose from the bottom of the beaker and floated on the surface of the medium, or floating lag time (FLT), and the duration the tablet remained floating on the surface of the medium, or total floating time (TFT), were determined by visual observation of its floating behavior in $0.1 \mathrm{~N} \mathrm{HCl}$ solution at $37 \pm 0.5{ }^{\circ} \mathrm{C}[20]$ using dissolution tester apparatus (VK7000, Vankel, US).

\section{In vitro drug release}

Drug release studies of the prepared floating tablets were carried out using the paddle method (apparatus type II) at 50 rotations per min by dissolution tester apparatus. Each tablet was placed into $900 \mathrm{ml}$ of $0.1 \mathrm{~N}$ $\mathrm{HCl}$ solution with the maintained temperature at $37 \pm 0.5^{\circ} \mathrm{C}$. The sample solution was taken every hour from the vessel in the volume of $10 \mathrm{ml}$, and then filtered through a $0.45 \mu \mathrm{m}$ filter. The amount of drug release in each sample was determined at a wavelength of $276 \mathrm{~nm}$ using a UVVisible spectrophotometer. The withdrawn amount $(10 \mathrm{ml})$ of each sample solution was replaced with the fresh medium $[6,19]$. 


\section{Molecular interaction}

\section{Thermal study}

Differential scanning calorimetry (DSC) curves of the sample were determined using a differential scanning calorimeter (DSC822, Mettler Toledo, Switzerland). Mass of the sample (2-3 mg) was weighed accurately and placed into $40-\mu \mathrm{l}$ open aluminum pan. The sample was heated at a temperature ranging between $25-320^{\circ} \mathrm{C}$ at a heating rate of $10^{\circ} \mathrm{C} / \mathrm{min}$ under a constant flow of nitrogen gas.

\section{Fourier transform infrared (FTIR) spectroscopy}

Fourier transform infrared (FTIR) spectra of the sample were determined using an FTIR spectrophotometer (Spectrum One, Perkin Elmer, Norwalk, CT). The sample was gently titrated and mixed with potassium bromide in a weight ratio of $1: 100$, and then compressed using a hydrostatic press at a pressure of 10 tons for 5 min. The disc was placed in the sample holder and scanned from 4000 to 600 per $\mathrm{cm}$ at a resolution of 4 per $\mathrm{cm}$.

\section{Statistical analysis}

The statistical analysis was performed using the SPSS program for Microsoft Windows, release 19 (SPSS (Thailand) Co. Ltd., Bangkok, Thailand). The results were expressed as the mean \pm SD. One-way ANOVA and independent samples t-test were used to test the statistical significance of differences among groups. The significance was determined with $95 \%$ confident limits $(\alpha=0.5)$ and was considered significant at a level of P less than 0.05 .

\section{RESULTS AND DISCUSSION}

\section{DICL solubility}

The effects of SLS ( 1 and $2 \%$ in $0.1 \mathrm{~N} \mathrm{HCl} \mathrm{solution)} \mathrm{on} \mathrm{DICL} \mathrm{solubility}$ of DICL were determined. The solubility of DICL in $0.1 \mathrm{~N} \mathrm{HCl}$ solution was $3.48 \pm 0.05 \mu \mathrm{g} / \mathrm{ml}$. Addition of SLS significantly increased the solubility of DICL in $0.1 \mathrm{~N} \mathrm{HCl}(54.24 \pm 4.76$ and $114.18 \pm 15.41 \mu \mathrm{g} / \mathrm{ml}$ for 1 and $2 \%$ SLS, respectively) $\left({ }^{*} \mathrm{P}<0.05\right)$. The improvements in solubility were significantly pronounced at higher concentrations of SLS $\left({ }^{*} \mathrm{P}<0.05\right)$. Unfortunately, in the preliminary study, it was found that the addition of SLS in the release medium had a negative effect on the in vitro buoyancy property. The prepared DICL floating tablets could not float in the medium containing SLS. These might relate to the density and surface active properties of the medium containing SLS. Therefore, in order to study the effect of tablet compositions on the in vitro buoyancy and release, $0.1 \mathrm{~N} \mathrm{HCl}$ solution was used in the study. Additionally, in order to distinguish the release profiles between different formulations, non-sink conditions might be appropriate, as the release testing under sink conditions generally leads to rapid release rates. It was claimed that the release testing under non-sink conditions can be a predictive tool for formulation development as well as batch-to-batch quality control [21].

Table 2: Weight variation, thickness, hardness, friability and drug content of DICL floating tablets

\begin{tabular}{|c|c|c|c|c|c|}
\hline Formulation & Weight variation (mg) ${ }^{a}$ & Thickness (mm) ${ }^{b}$ & Hardness $(\mathrm{kg} / \mathrm{cm} 2)^{\mathrm{b}}$ & Friability (\%) & Drug content $(\%)^{\mathrm{c}}$ \\
\hline F1 & $250.1 \pm 0.2$ & $3.6 \pm 0.0$ & $8.3 \pm 0.1$ & 0.47 & $102.5 \pm 0.3$ \\
\hline $\mathrm{F} 2$ & $250.0 \pm 0.3$ & $3.6 \pm 0.0$ & $8.4 \pm 0.2$ & 0.13 & $104.5 \pm 0.1$ \\
\hline F3 & $250.0 \pm 0.1$ & $3.6 \pm 0.0$ & $8.2 \pm 0.1$ & 0.36 & $104.0 \pm 0.1$ \\
\hline $\mathrm{F} 4$ & $250.0 \pm 0.2$ & $3.6 \pm 0.0$ & $8.4 \pm 0.1$ & 0.45 & $102.2 \pm 0.1$ \\
\hline F5 & $250.6 \pm 0.4$ & $3.6 \pm 0.0$ & $8.5 \pm 0.2$ & 0.30 & $103.7 \pm 0.5$ \\
\hline F6 & $250.2 \pm 0.2$ & $3.6 \pm 0.0$ & $8.5 \pm 0.3$ & 0.36 & $100.5 \pm 0.3$ \\
\hline F7 & $250.0 \pm 0.2$ & $3.6 \pm 0.0$ & $8.6 \pm 0.2$ & 0.31 & $100.7 \pm 0.4$ \\
\hline FS1 & $249.4 \pm 0.3$ & $3.6 \pm 0.0$ & $8.2 \pm 0.2$ & 0.51 & $104.0 \pm 0.2$ \\
\hline FS2 & $249.8 \pm 0.2$ & $3.6 \pm 0.0$ & $8.5 \pm 0.3$ & 0.41 & $100.6 \pm 0.1$ \\
\hline FS3 & $250.0 \pm 0.4$ & $3.6 \pm 0.0$ & $8.4 \pm 0.2$ & 0.41 & $101.3 \pm 0.1$ \\
\hline
\end{tabular}

${ }^{\mathrm{a}}$ mean $\pm \mathrm{SD}, \mathrm{n}=20$, ${ }^{\mathrm{b}}$ mean $\pm \mathrm{SD}, \mathrm{n}=6$, ${ }^{\mathrm{c}}$ mean $\pm \mathrm{SD}, \mathrm{n}=3$.

\section{Tablet properties}

All DICL tablets of the floating formulations were evaluated for weight variation, thickness, hardness, friability, and drug content. As shown in table 2, the average weight of the tablets ranged between 249.4 and $250.6 \mathrm{mg}$ which was within the range of the limitation criteria of $\pm 5 \%$. All formulations showed the uniform thickness of $3.6 \mathrm{~mm}$. The hardness of the tablets ranged between 8.2 and 8.6 $\mathrm{kg} / \mathrm{cm} 2$ for all formulations, with friability of less than $1 \%$. The drug content was within the acceptable ranges of $90-110 \%$ for all formulations. These results were found to be satisfactory and within the acceptance criteria in USP 39-NF 34 [18].

\section{Effects of gas-forming agent type}

The effects of each type of gas-forming agents, namely $\mathrm{CaCO}_{3}$ and $\mathrm{NaHCO}_{3}$ (F1 and F2, respectively) on swelling index, in vitro buoyancy and release properties were shown in table 3 and fig. 1 . The determination of the swelling index of the tablets was performed in order to determine the swelling capacity of the tablets in $0.1 \mathrm{~N} \mathrm{HCl}$ solution at $37 \pm 0.5{ }^{\circ} \mathrm{C}$ for $3 \mathrm{~h}$. The swelling index was plotted against time as seen in fig. 1a. It could be seen that the swelling profile obtained from DICL floating tablets containing $\mathrm{NaHCO}_{3}$ was higher than that containing $\mathrm{CaCO}_{3}$. The initial swelling rate, calculated over the study time range of $0-0.5 \mathrm{~h}$, of DICL floating tablets containing $\mathrm{NaHCO}_{3}$ was significantly higher than that of the tablets containing $\mathrm{CaCO}_{3}\left({ }^{*} \mathrm{P}<0.05\right)$. Moreover, the swelling index of the tablets containing $\mathrm{NaHCO}_{3}$ measured at $3 \mathrm{~h}$ was significantly higher than that of the tablets containing $\mathrm{CaCO}_{3}\left({ }^{*} \mathrm{P}<0.05\right)$. This may be due to the higher aqueous solubility and higher gas-forming efficiency of $\mathrm{NaHCO}_{3}$, which generated more pores inside the tablet matrix, leading to faster water uptake and higher swelling capacity when compared to $\mathrm{CaCO}_{3}$ [22].

The buoyancy properties, consisting of floating lag time (FLT) and total floating time (TFT), of each DICL floating formulation, were determined. FLT is defined as the duration the dosage form rises to the surface of the medium, while TFT is defined as the total duration the dosage constantly remains floating. The in vitro buoyancy results were shown in fig. 1b. It could be seen that the tablets containing $\mathrm{NaHCO}_{3}$ showed significantly shorter FLT but longer TFT compared to those of the tablets containing $\mathrm{CaCO}_{3}\left({ }^{*} \mathrm{P}<0.05\right)$. These results indicated that $\mathrm{NaHCO}_{3}$ provided better buoyancy properties compared to $\mathrm{CaCO}_{3}$. Such an outcome was in line with the previous work of ciprofloxacin and cinnarizine $\mathrm{HCl}$ floating tablets $[6,22]$. The buoyancy capability of tablets may be described using equation 3 [23]. The total force acting vertically on the immersed object, $F$, that was given by the vectorial sum of buoyancy, $F_{(b)}$, and gravitational forces, $F_{(\mathrm{g})}$, acting on the test object can be expressed below.

$$
F=F_{(\mathrm{b})}-F_{(\mathrm{g})}=\left(d_{\mathrm{f}}-d_{\mathrm{s}}\right) g V
$$

Where $d_{\mathrm{f}}$ and $d_{\mathrm{s}}$ represent the fluid density and solid object density, $g$ is the acceleration due to gravity and $W$ and $V$ are the weight and volume of the test objects. The better floating is exhibited by the object when $F$ value is more positive $[23,24]$. According to equation $3, \mathrm{NaHCO}_{3}$ generated greater gas and pores inside tablets, which resulted in lower density, $d_{\mathrm{s}}$, of tablets compared to $\mathrm{CaCO}_{3}$. Therefore, $\mathrm{NaHCO}_{3}$ exhibited better buoyancy capacity when compared to $\mathrm{CaCO}_{3}$.

The in vitro release study was conducted to investigate the effects of type of gas-forming agent on the release-time profiles of DICL from the tablets. The typical release-time profiles are shown in fig. 1c. It 
clearly shows that DICL release from tablets containing $\mathrm{NaHCO}_{3}$ was higher than that of the tablets containing $\mathrm{CaCO}_{3}$. The initial release rates, calculated over the study time range of $0-1 \mathrm{~h}$, of DICL floating tablets containing $\mathrm{NaHCO}_{3}$ was significantly higher than that of the tablets containing $\mathrm{CaCO}_{3}\left({ }^{*} \mathrm{P}<0.05\right)$. At $12 \mathrm{~h}$, the cumulative release obtained from tablets containing $\mathrm{NaHCO}_{3}$ was significantly higher than that of the tablets containing $\mathrm{CaCO}_{3}\left({ }^{*} \mathrm{P}<0.05\right)$. The initial release rate within the first hour was found to be in the same order $\left(\mathrm{NaHCO}_{3}>\mathrm{CaCO}_{3}\right)$ as the swelling index at $3 \mathrm{~h}$, but the opposite order was noted for the FLT of the tablets. The concentration of DICL in 0.1 $\mathrm{N} \mathrm{HCl}$ medium at $12 \mathrm{~h}$ from the tablets containing $\mathrm{NaHCO}_{3}$ was higher than that from the tablets containing $\mathrm{CaCO}_{3}(5.95 \pm 0.02$ and $4.15 \pm 0.03 \mu \mathrm{g} / \mathrm{ml}$, respectively). The solubility of both concentrations was significantly higher than that of DICL in medium $\left({ }^{*} \mathrm{P}<0.05\right)$. This may be due to the higher efficacy of $\mathrm{NaHCO}_{3}$ as an alkalizing agent in increasing the tablet microenvironment $\mathrm{pH}$ compared to $\mathrm{CaCO}_{3}$.

The tablets with higher swelling capacity but short FLT tended to give the higher initial release rate and the higher release-time profile. The presence of $\mathrm{NaHCO}_{3}$ might have been responsible for this situation because of its higher gas-forming capacity, higher aqueous solubility of $\mathrm{NaHCO}_{3}[22]$ and higher alkalizing property compared to $\mathrm{CaCO}_{3}$. As a result, $\mathrm{NaHCO}_{3}$ was chosen as a gas-forming agent for further studies.

Table 3: Effects of formulation parameters on swelling, in vitro buoyancy and release properties of DICL floating tablets $(\mathrm{mean} \pm \mathrm{SD}, \mathrm{n}=3$ )

\begin{tabular}{llllll}
\hline Formulation & Swelling properties & & \multicolumn{2}{c}{ Buoyancy properties } & Release properties \\
\cline { 2 - 6 } & $\begin{array}{l}\text { Initial swelling rate } \\
(\% / \mathbf{h})\end{array}$ & $\begin{array}{l}\text { Swelling index at 3 } \\
\text { h (\%) }\end{array}$ & FLT (min) & TFT (h) & $\begin{array}{l}\text { Initial release rate } \\
\text { (\%)/h) }\end{array}$ \\
\hline F1 & $115 \pm 11$ & $144 \pm 11$ & $10.04 \pm 0.04$ & $10.0 \pm 0.0$ & $0.95 \pm 0.05$ \\
F2 & $1412 \pm 25$ & $1074 \pm 16$ & $0.39 \pm 0.03$ & $>12$ & $1.67 \pm 0.02$ \\
F3 $(\%)$
\end{tabular}

FLT: floating lag time, TFT: total floating time, ${ }^{*}$ not applicable.

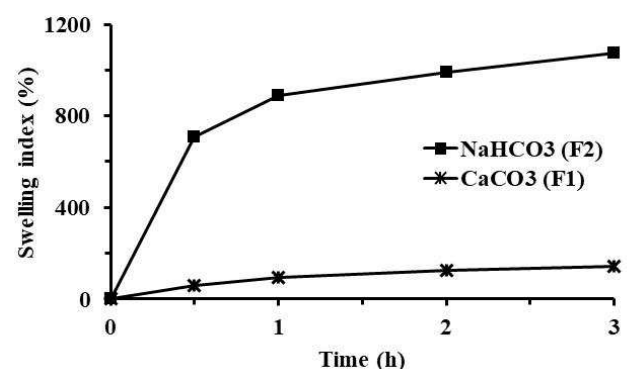

(a)

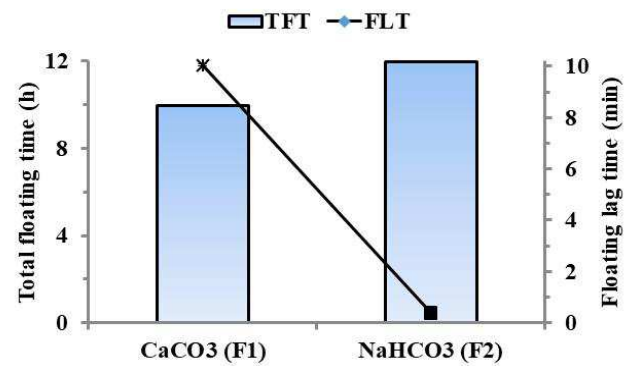

(b)

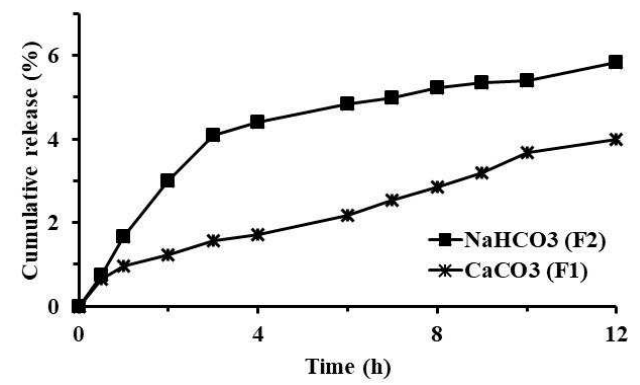

(c)

Fig. 1: Effects of gas-forming agent type including $\mathrm{CaCO}_{3}$ and $\mathrm{NaHCO}_{3}$ on swelling index (a), in vitro buoyancy (b) and in vitro release of DICL floating tablets (mean $\pm S D, n=3$ )

\section{Effects of $\mathrm{NaHCO}_{3}$ concentration}

The effects of concentrations of $\mathrm{NaHCO}_{3}$ at 10, 20 and 30\% (F3, F2 and $\mathrm{F} 4$, respectively) on swelling index, in vitro buoyancy and release were shown in table 3 and fig. 2 . It could be seen in fig. 2a that the tablets containing 10 and $20 \% \mathrm{NaHCO}_{3}$ showed a comparable swelling profile that was higher compared to the tablets containing 30\% $\mathrm{NaHCO}_{3}$. Surprisingly, the increases of $\mathrm{NaHCO}_{3}$ concentration from 10 to 20 and $30 \%$ resulted in the significant decreases of initial swelling rates of DICL floating tablets $\left({ }^{*} \mathrm{P}<0.05\right)$. $\mathrm{NaHCO}_{3}$ is freely soluble in water and able to absorb water rapidly [25]. The increase of $\mathrm{NaHCO}_{3}$ might enhance the ability of $\mathrm{NaHCO}_{3}$ to impede water uptake and initial swelling of tablets. However, the swelling index at $3 \mathrm{~h}$, obtained from the tablets containing 10 and $20 \% \mathrm{NaHCO}_{3}$, was comparable $(\mathrm{P}>0.05)$. These findings are parallel to those of the previous work which reported that the change of $\mathrm{NaHCO}_{3}$ concentration had an insignificant effect on the swelling of tablets [26]. It should be noted that the swollen tablets containing $30 \% \mathrm{NaHCO}_{3}$ could not be completely taken out for evaluation after $1 \mathrm{~h}$. This may be because the high amount of $\mathrm{NaHCO}_{3}$ generated too much gas bubbles and pore formation in tablets, leading to excessively high porosity and delicacy.

The in vitro buoyancy properties of tablets containing different amounts of $\mathrm{NaHCO}_{3}$ were shown in fig $2 \mathrm{~b}$. It could be seen that the tablets containing 20 and $30 \% \mathrm{NaHCO}_{3}$ provided comparable short FLT ( $<1 \mathrm{~min})(\mathrm{P}>0.05)$, which was shorter than that of the tablets containing $10 \% \mathrm{NaHCO}_{3}\left({ }^{*} \mathrm{P}<0.05\right)$. All tablets containing different amounts of $\mathrm{NaHCO}_{3}$ could be floated for at least $12 \mathrm{~h}$. This finding was similar to that of the previous works $[2,6,19]$. This study revealed that the higher amount of $\mathrm{NaHCO}_{3}(20$ and $30 \%)$ could enhance the gas-forming rate, and the high amount of $\mathrm{NaHCO}_{3}$ inside the tablets resulted in their shorter FLT. Furthermore, $20 \% \mathrm{NaHCO}_{3}$ might be enough to shorten the FLT of DICL floating tablets.

The effects of the amounts of $\mathrm{NaHCO}_{3}$ (at 20 and 30\%) on the release of DICL floating tablets were determined and shown in fig. 2c. The effect on tablets containing $10 \% \mathrm{NaHCO}_{3}$ was not determined because their FLT was too long. It could be seen that DICL release from the tablets containing $30 \% \mathrm{NaHCO}_{3}$ was higher than that of the tablets containing $20 \% \mathrm{NaHCO}_{3}$. The initial release rate of DICL floating tablets containing $30 \% \mathrm{NaHCO}_{3}$ was significantly higher than that of the tablets containing $20 \% \mathrm{NaHCO}_{3}\left({ }^{*} \mathrm{P}<0.05\right)$. At $12 \mathrm{~h}$, the cumulative release obtained from the tablets containing $30 \%$ $\mathrm{NaHCO}_{3}$ was significantly higher than that of the tablets containing 
$20 \% \mathrm{NaHCO}_{3}\left({ }^{*} \mathrm{P}<0.05\right)$. This is parallel to the previous works which reported that the increase in the concentration of gas-forming agents resulted in the higher drug release $[6,20]$. The concentration of DICL released at $12 \mathrm{~h}$ from the tablets containing $30 \% \mathrm{NaHCO}_{3}$ was higher than that from the tablets containing $20 \% \mathrm{NaHCO}_{3}$ $(6.67 \pm 0.05$ and $5.95 \pm 0.02 \mu \mathrm{g} / \mathrm{ml}$, respectively) and the solubility of DICL in medium, respectively. The increase of $\mathrm{NaHCO}_{3}$ from 20 to $30 \%$ might increase the hydrophilicity and wettability of the tablets and the excess amount of $\mathrm{NaHCO}_{3}$ might raise the microenvironment $\mathrm{pH}$, resulting in an increase of DICL solubility.

It has been reported that the increase of $\mathrm{NaHCO}_{3}$ concentration in floating tablets resulted in the worsen flowability of powder [19]. Furthermore, the increase of $\mathrm{NaHCO}_{3}$ from 20 to $30 \%$ had no effect on the buoyancy properties but was likely to improve the friability of floating tablets. Therefore, $\mathrm{NaHCO}_{3}$ at $20 \%$ was chosen to be used in further studies.

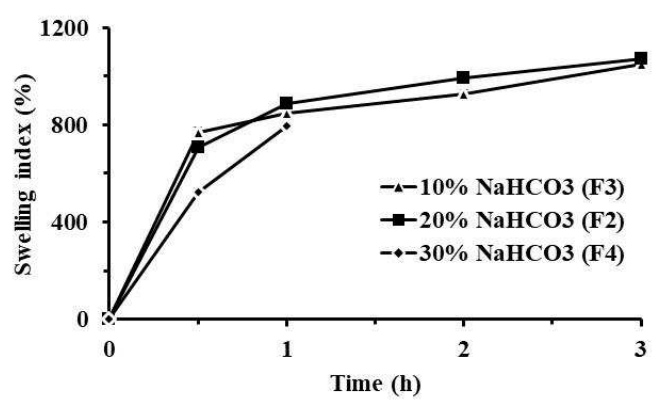

(a)

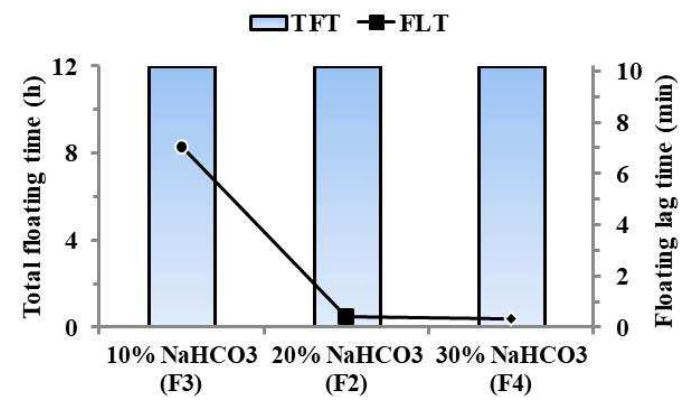

(b)

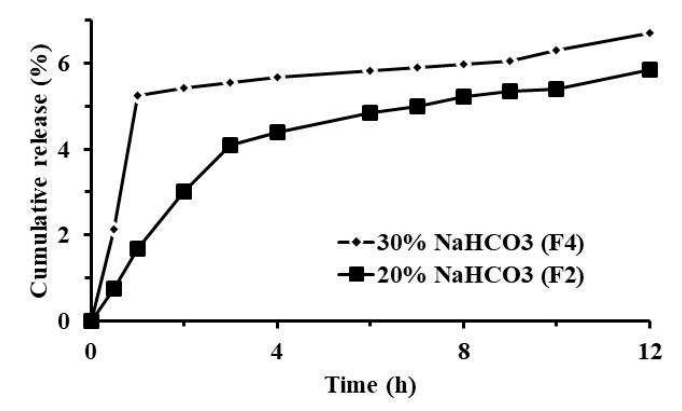

(c)

Fig. 2: Effects of $\mathrm{NaHCO}_{3}$ concentration on the swelling index (a), in vitro buoyancy (b) and in vitro release of DICL floating tablets (mean $\pm S D, n=3)$

\section{Effects of hydroxypropyl methylcellulose (HPMC) grade}

Hydroxypropyl methylcellulose (HPMC) is one of the cellulose ether polymers that have been widely used as floating matrices and swelling polymer in the floating dosage forms [27]. There are many grades of HPMC available in the market. HPMC K15M and K100M, which were different in terms of molecular weight and solution viscosity, were chosen for the study on the effects of HPMC grades. The effects of different grades of HPMC, namely HPMC K15M and K100M (F7 and F2, respectively), on swelling index, in vitro buoyancy and release of DICL floating tablets were shown in table 3 and fig. 3. It could be seen in fig. 3a that the swelling profile and the swelling index at $3 \mathrm{~h}$ obtained from the tablets containing HPMC K100M were significantly higher than those of the tablets containing HPMC K15M $(* \mathrm{P}<0.05)$. The result was in line with that of the previous studies $[26,28]$ which reported that the higher swelling capacity was obtained with a higher grade of HPMC. HPMC $\mathrm{K} 100 \mathrm{M}$ and $\mathrm{K} 15 \mathrm{M}$ are different in terms of molecular weight, water uptake and solution viscosity. It is known that the ability of swelling formation is dependent on the presence of hydrophilic groups. The water uptake of these functional groups results in water entry into polymer network, leading to expansion and consequently an ordering of polymer chains. The swelling equilibrium (maximum water uptake) is reached when the osmotic forces of the functional groups are balanced by the restrictive forces of the higher ordering of the polymer chains [29, 30]. The HPMC K100M may enhance greater water uptake and expansion of polymer chains compared to HPMC K15M.

The effects of HPMC grade on buoyancy properties of DICL floating tablets were shown in fig. $3 \mathrm{~b}$. It was noted that the tablets containing HPMC K15M and K100M showed comparable FLT and TFT ( $\left.{ }^{*} \mathrm{P}>0.05\right)$. The result was in line with that of the previous work [31] which reported the comparable effects of different molecular weights of chitosan on FLT and TFT of acetylsalicylic acid floating tablets. According to equation 3 and swelling index results, the tablets containing HPMC K100M might have greater volume, $V$, and density $d_{s}$, than those of the tablets containing HPMC K15M during the buoyancy study. As a result, the comparable buoyancy capability was observed.

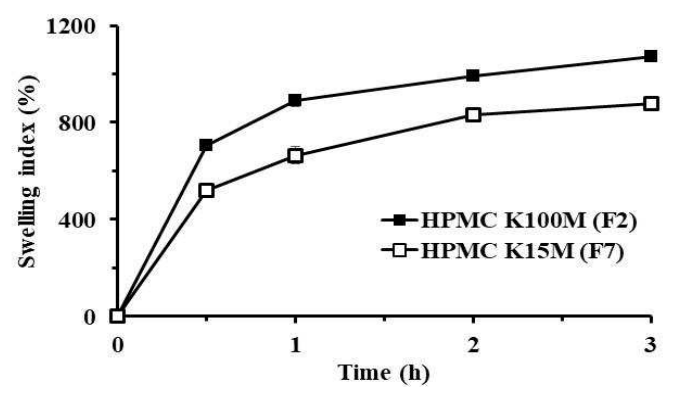

(a)

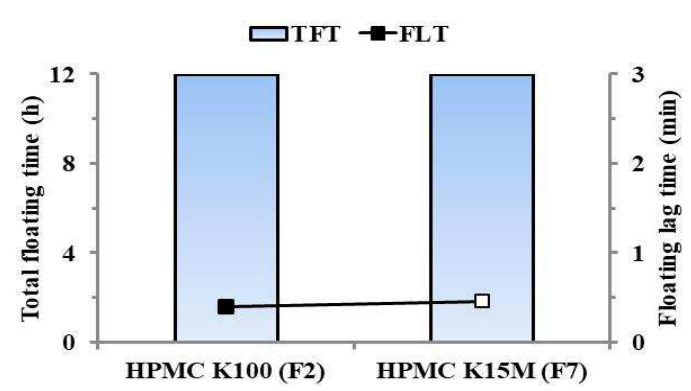

(b)

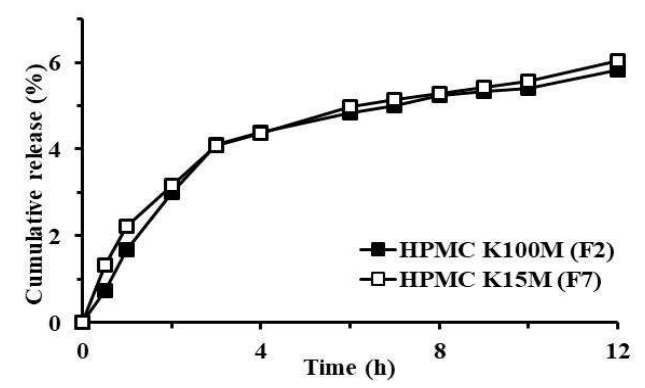

(c)

Fig. 3: Effects of HPMC grades, namely HPMC K15M and K100M, on the swelling index (a), in vitro buoyancy (b) and in vitro release of DICL floating tablets (mean $\pm S D, n=3$ ) 
The effect of HPMC grade investigated in the in vitro release study was shown in fig. 3c. It could be seen that the DICL releases from the tablets containing HPMC K15M and $100 \mathrm{M}$ were comparable. The initial release rates and the cumulative releases at $12 \mathrm{~h}$ obtained from the tablets containing HPMC K15M and $100 \mathrm{M}$ were comparable $(\mathrm{P}>0.05)$. The concentrations of DICL in the medium at $12 \mathrm{~h}$ from the tablets containing $\mathrm{K} 15 \mathrm{M}$ and $100 \mathrm{M}$ were also comparable $(5.97 \pm 0.02$ and $5.95 \pm 0.02 \mu \mathrm{g} / \mathrm{ml}$, respectively).

This study revealed that HPMC grade affected only the swelling properties of tablets. However, it had no effect on the buoyancy capability and DICL release of the tablets containing HPMC. As a result, HPMC K100M was chosen as the representative of HPMC in further studies.

\section{Effects of HPMC concentration}

The effects of concentrations of HPMC K100M at 10, 20 and 30\% (F5, F2 and F6, respectively) on swelling index, in vitro buoyancy and release of DICL floating tablets, were shown in table 3 and fig. 4 . It could be seen in fig. 4a that the increase of HPMC concentration from 10 to $20 \%$ resulted in the higher swelling profile and initial swelling rate $\left({ }^{*} \mathrm{P}<0.05\right)$. However, the increase of HPMC concentration from 20 to $30 \%$ resulted in lower initial part of the swelling profile and initial swelling rate $\left({ }^{*} \mathrm{P}<0.05\right)$. However, at $3 \mathrm{~h}$, a swelling index of tablets increased when the concentration of HPMC increased from 10 to $30 \%$ $\left({ }^{*} \mathrm{P}<0.05\right)$. This result was similar to that of the previous study, which reported that when the concentration of polymers was higher, the swelling properties of the tablets increased as a result [32]. In the case of $30 \%$ HPMC, the high amount of HPMC may impede the initial water uptake and in turn, the swelling of the polymer.

The in vitro buoyancy results were shown in fig. $4 \mathrm{~b}$. It could be seen that the tablets containing 10 and $20 \%$ HPMC showed comparable FLT $(\mathrm{P}>0.05)$, which were shorter than that observed in the tablets containing $30 \%$ HPMC $(* \mathrm{P}<0.05)$. All tablets containing various concentrations of HPMC could be floated for at least $12 \mathrm{~h}$. This result was in line with that of the previous work [33] which reported that the increase of HPMC concentration was likely to prolong FLT. This may be because the high concentration of HPMC inhibited the initial hydration of medium into the matrix of the tablets, then retarded the gas forming, resulting in the prolonged FLT.

The effect of HPMC concentration on the release of DICL floating tablets was determined and shown in fig 4c. It could be seen that DICL release from the tablets containing 10\% HPMC was higher than those from the tablets containing 20 and 30\% HPMC. The initial release rates and the cumulative releases obtained from the tablets containing 10\% HPMC were significantly higher than those from the tablets containing 20 and $30 \%$ HPMC $(* \mathrm{P}<0.05)$. The concentration of DICL released at $12 \mathrm{~h}$ from the tablets containing 10\% HPMC K100M was higher than those from the tablets containing 20 and 30\% HPMC K100M $(6.27 \pm 0.02,5.95 \pm 0.02$ and $5.73 \pm 0.03 \mu \mathrm{g} / \mathrm{ml}$, respectively). This was because the higher polymer molecular weight or higher polymer concentration formed the higher strength gel-swellable structure, which retarded the release of drug from the matrix $[34,35]$, and may also retard the release and interaction of $\mathrm{NaHCO} 3$ with $0.1 \mathrm{~N} \mathrm{HCl}$ solution.

The tablets with a lower concentration of HPMC K100 were likely to shorten FLT and increase DICL release. Therefore, 10 and 20\% HPMC were chosen for further study of SLS effects.

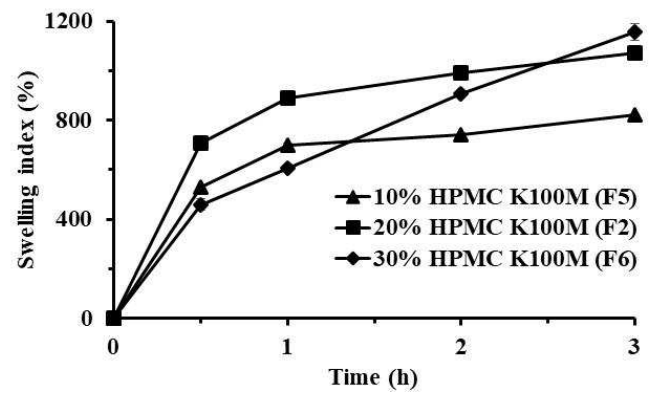

(a)

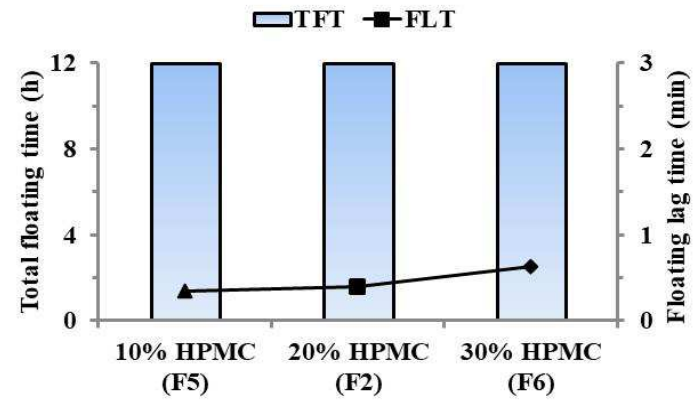

(b)

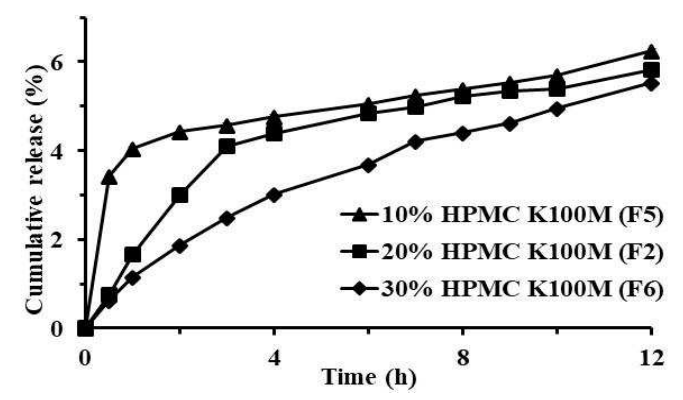

(c)

Fig. 4: Effects of HPMC K100M concentration on swelling index (a), in vitro buoyancy (b) and in vitro release of DICL floating tablets (mean $\pm S D, n=3$ )

\section{Effects of SLS concentration}

The limited amount of DICL release from DICL floating tablets was due to the poor solubility of DICL in $0.1 \mathrm{~N} \mathrm{HCl}$ solution. In an attempt to increase the DICL release, the SLS was incorporated as a wetting agent in the DICL floating tablets. The effects of concentrations of SLS at $0.25,0.5$ and $1 \%$ on swelling index, in vitro buoyancy and release of DICL floating tablets prepared with 10 or $20 \%$ HPMC K100M were determined and shown in table 3 and fig. 5 .

In the case of DICL floating tablets containing 10\% HPMC K100M, it was found that the tablets swelled very fast and the swollen tablets could not be completely taken out for evaluation because of their high porosity and delicacy. For the buoyancy studies, the tablets containing 0.25 and $0.5 \%$ SLS showed comparable FLT, which was comparable to tablets without SLS ( $P>0.05$ ) (data not shown). They showed TFT of less than $1 \mathrm{~min}$. DICL floating tablets containing $1 \%$ SLS did not float. As a result, the in vitro release of DICL floating tablets containing 10\% HPMC K100M was not determined. This may be because SLS improved the wettability of the tablets and the exceptionally low concentration of HPMC remaining as floating matrix would lead to the loss of physical integrity.

In the case of DICL floating tablets containing 20\% HPMC K100M, it could be seen in fig. 5 a that all tablets with various concentrations of SLS were unable to exhibit complete swelling profile. The tablets containing 0.25 and $0.5 \%$ SLS (FS1 and FS2, respectively) could be evaluated only for a period of $0.5 \mathrm{~h}$, and they could not be completely taken out for evaluation after that. The increase of SLS concentration from 0.25 to $0.5 \%$ resulted in the significant increases of initial swelling rates of DICL floating tablets $\left({ }^{*} \mathrm{P}<0.05\right)$. When compared to the tablets without SLS, the tablets containing $0.25 \%$ SLS showed comparable initial swelling rates $(\mathrm{P}>0.05)$, but the tablets containing $0.5 \%$ SLS showed significantly higher initial swelling rate $\left({ }^{*} \mathrm{P}<0.05\right)$. For $1 \%$ SLS, the tablets swelled very fast, and the swollen tablets could not be completely taken out for evaluation. Incorporation of SLS at high concentrations (0.5 and 1\%) might increase the wettability of tablets, [16] which could result in the higher initial swelling rates.

The in vitro buoyancy results are shown in fig. $5 \mathrm{~b}$. It could be seen that all tablets containing various concentrations of SLS showed FLT 
of shorter than 1 min. The tablets containing 0.25 and $0.5 \%$ SLS showed comparable FLT $(\mathrm{P}>0.05)$, which were shorter than that of the tablets containing $1 \%$ SLS $\left({ }^{*} \mathrm{P}<0.05\right)$. When compared to the tablets without SLS, only the tablets containing $1 \%$ SLS showed significantly longer FLT $\left({ }^{*} \mathrm{P}<0.05\right)$. For the TFT, the tablets containing $0.25 \%$ SLS floated longer than those containing 0.5 and $1 \%$ SLS. It should be noted that all tablets containing various concentrations of SLS showed TFT of less than $12 \mathrm{~h}$. The increase of SLS concentration was likely to prolong the FLT but shorten the TFT. It is known that SLS has the wettability and disintegration properties. Incorporation of SLS into tablets at high concentrations ( 0.5 and $1 \%$ ) might increase the wettability and the ability to disintegrate the tablets, but decrease the interfacial tension between the tablets and the release medium. After the tablets came into contact with the release medium, the tablets might be wet quickly, followed by disaggregation of the tablets with less swelling of polymer, which would result in increases of $V$ and $d_{s}$, according to equation 3 and the swelling study.

Tablets containing $0.25 \%$ SLS were selected as representatives for observation of SLS effects. The effects of SLS incorporation at $0.25 \%$ on the release of DICL floating tablets were determined and shown in fig. 5c. It could be seen that the release of the tablets containing $0.25 \%$ SLS was higher than that of the tablets without SLS. The initial release rate of DICL floating tablets containing $0.25 \%$ SLS was higher than that of the tablets without SLS. At $12 \mathrm{~h}$, the cumulative release obtained from the tablets containing $0.25 \%$ SLS was significantly higher than that of the tablets without SLS $\left({ }^{*} \mathrm{P}<0.05\right)$. However, the integrity of these tablets did not remain after $0.5 \mathrm{~h}$. It clearly showed that the higher drug release was obtained with increased SLS concentration. This was due to the solubilization effect of SLS, together with the improvement of a hydrophilic environment and wettability of the tablets, which consequently enabled the higher drug release $[17,36]$.

The presence of SLS in DICL floating tablets were found to affect the swelling properties, in vitro buoyancy and release. Nevertheless, SLS could not be used in DICL floating tablets in this study.

\section{Molecular interactions}

Molecular interactions between DICL and each excipient in DICL floating tablets were investigated using DSC and FTIR spectroscopy.

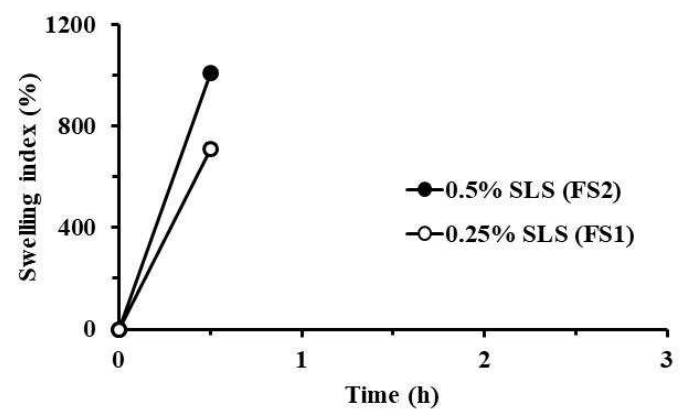

(a)

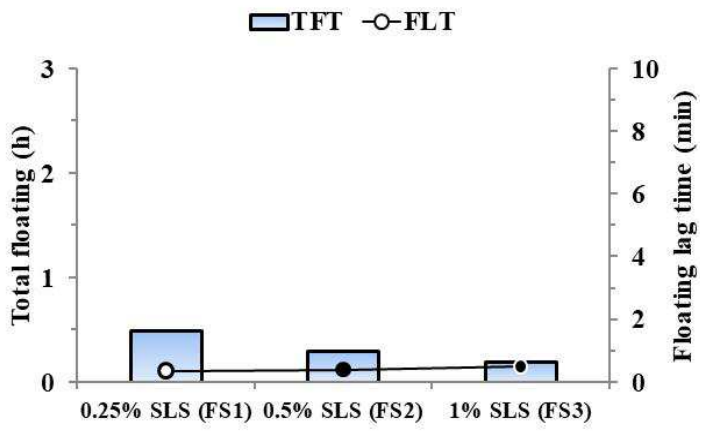

(b)

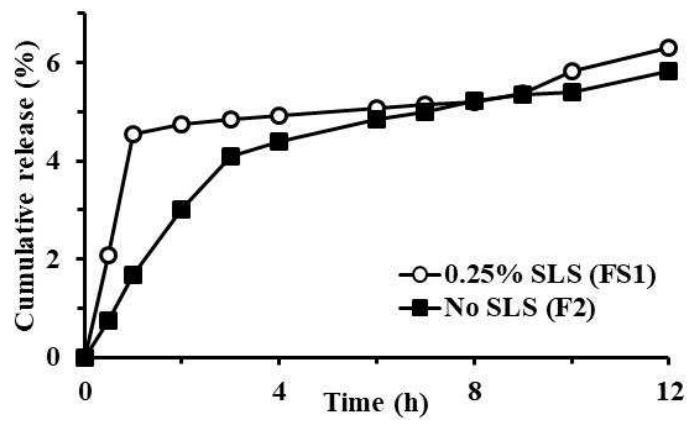

(c)

Fig. 5: Effects of SLS on swelling index (a), in vitro buoyancy (b) and in vitro release of DICL floating tablets containing $20 \%$ HPMC K100M (mean $\pm S D, n=3$ )

\section{Thermal study}

The DSC thermograms of DICL, each excipient $\left(\mathrm{NaHCO}_{3}\right.$, Aerosil, MCC, Mg stearate, SLS and HPMC K100M) and the mixture of DICL with each excipient (at a ratio of 1:1) are presented in fig. 6. A sharp exothermic peak appeared at $284.0^{\circ} \mathrm{C}$ (fig. 6a), corresponding to the melting point of DICL $\left(283-285^{\circ} \mathrm{C}\right)$ [37]. As presented in fig. 6c, 6e and $6 \mathrm{~g}$, there was no change in the exothermic peak of DICL in the mixture thermograms, exhibiting the compatibility of the drug with $\mathrm{NaHCO}_{3}$, Aerosil and MCC, respectively. The DSC thermogram of DICL-Mg stearate (fig. 6i) became broaden due to the evaporation of Mg stearate, which had a flash point of $250^{\circ} \mathrm{C}$ [25]. As shown in fig. $6 \mathrm{k}$, the DSC curve of DICL-SLS mixture almost disappeared due to the lower melting points (endothermal events) of SLS compared to DICL, and the decomposition phenomena of SLS at $192.6{ }^{\circ} \mathrm{C}$, suggesting that DICL might be dissolved in the melted SLS [38]. Fig. $6 \mathrm{~m}$ presented the DSC curve of DICL-HPMC K100M which exhibited the shift of the peak to lower temperature, which was possibly due to the high melting of HPMC K100 that interfered the DSC peak.

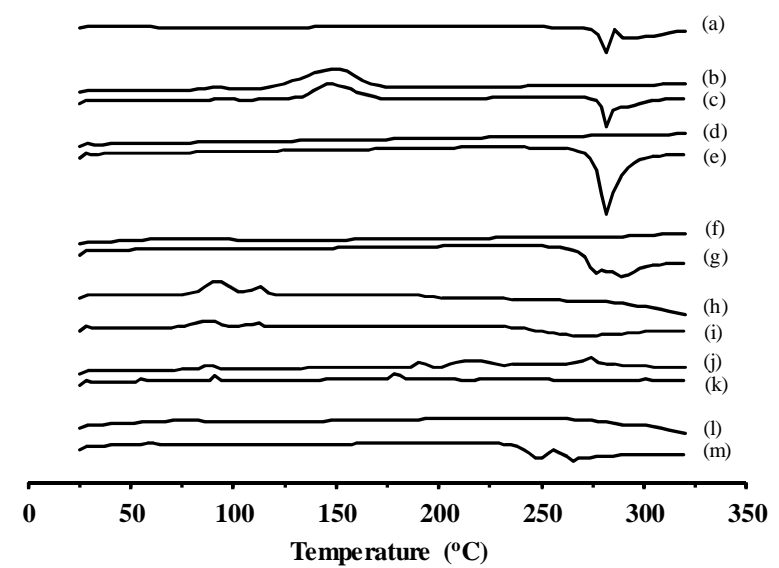

Fig. 6: DSC thermograms of DICL (a), $\mathrm{NaHCO}_{3}$ (b), DICL-NaHCO (c), Aerosil (d), DICL-Aerosil (e), MCC (f), DICL-MCC (g), Mg stearate (h), DICL-Mg stearate (i), SLS (j), DICL-SLS (k), HPMC K100M (l) and DICL-HPMC K100M (m)

\section{Fourier transform infrared (FTIR) spectroscopy}

The FTIR spectra of DICL, each major ingredient $\mathrm{NaHCO}_{3}, \mathrm{MCC}$ and HPMC K100M) and mixtures of DICL-each ingredient (at the ratio as F2) are shown in fig. 7. The distinct peaks of DICL at $3387 \mathrm{~cm}-1(\mathrm{NH}$ stretching of secondary amine), $1557 \mathrm{~cm}-1$ (C=C stretching), 1305 cm-1 (C-N stretching), and $747 \mathrm{~cm}-1$ (C-Cl stretching) [39] are shown in the spectra of DICL and DICL-excipients. Based on the FTIR results, it seemed that there was no interaction between DICL and the ingredients tested. 


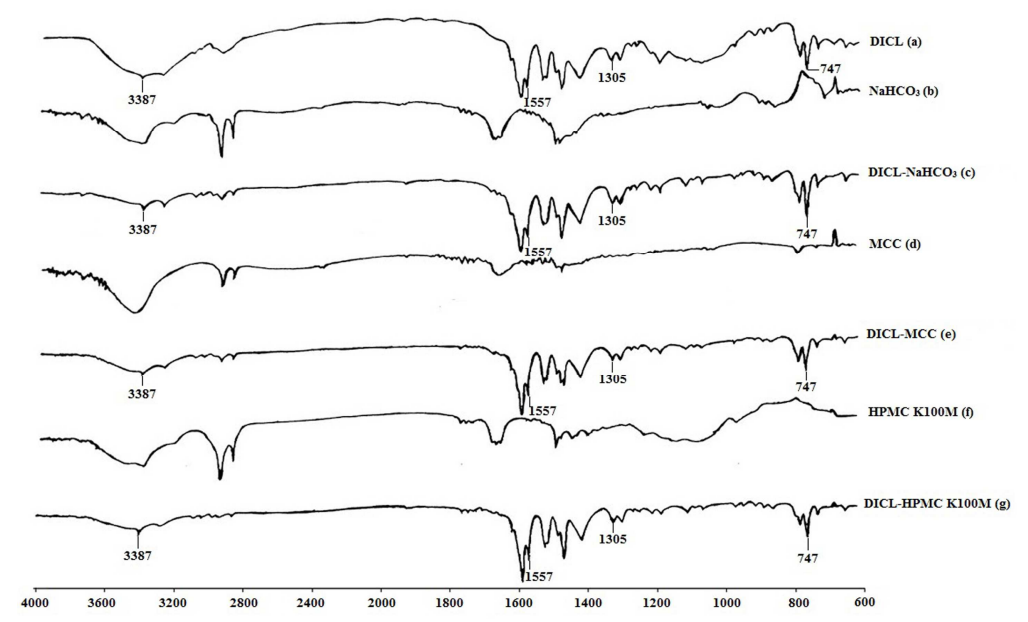

Fig. 7: FTIR spectra of DICL (a), $\mathrm{NaHCO}_{3}$ (b), DICL-NaHCO 3 (40:20) (c), MCC (d), DICL-MCC (40:18.5) (e), HPMC K100M (f) and DICL-HPMC K100M (40:20) (g)

\section{CONCLUSION}

In this study, DICL floating tablets were prepared successfully. The weight variation, thickness, hardness, friability, and drug content were satisfied and within the acceptance criteria of USP 39-NF 34. The effects of type and concentration of gas-forming agents, as well as grade and concentration of floating matrix and swelling polymers, were verified. The molecular interaction between DICL and excipients in the formulation was excluded. Incorporation of SLS as a wetting and solubilizing agent revealed the enhancing effects on the rate and cumulative amount of DICL releases from the floating tablets. Nevertheless, SLS addition showed unacceptable adverse effects on swelling and buoyancy properties of DICL floating tablets. SLS, therefore, could not be used as the wetting agent for DICL floating tablets.

The release testing under non-sink conditions was able to distinguish the effects of formulation parameters on the DICL release profiles. As a result, the limited release from the developed floating tablets was obtained. For realistic release and in vivo characterization, the release under sink conditions and/or in vivo pharmacokinetic study will be further carried out.

\section{ACKNOWLEDGMENT}

The authors wish to thank the Royal Scholarship under Her Royal Highness Princess Maha Chakri Sirindhorn Education Project to the Kingdom of Cambodia, the Center for Research and Development of Herbal Health Products and Faculty of Pharmaceutical Sciences, Khon Kaen University, Thailand.

\section{AUTHORS CONTRIBUTIONS}

All the authors have contributed equally

\section{CONFLICT OF INTERESTS}

The authors report no conflicts of interest

\section{REFERENCES}

1. Jagdale SC, Agavekar AJ, Pandya SV, Kuchekar BS, Chabukswar AR. Formulation and evaluation of gastroretentive drug delivery system of propranolol hydrochloride. AAPS PharmSciTech 2009;10:1071-9.

2. Yin L, Qin C, Chen K, Zhu C, Cao H, Zhou J, et al. Gastro-floating tablets of cephalexin: preparation and in vitro/in vivo evaluation. Int J Pharm 2013;452:241-8.

3. Singh BN, Kim KH. Floating drug delivery systems: an approach to oral controlled drug delivery via gastric retention. J Controlled Release 2000;63:235-59.

4. Lopes CM, Bettencourt C, Rossi A, Buttini F, Barata P. Overview on gastroretentive drug delivery systems for improving drug bioavailability. Int J Pharm 2016;510:144-58.
5. Bardonnet PL, Faivre V, Pugh WJ, Piffaretti JC, Falson F. Gastroretentive dosage forms: overview and special case of helicobacter pylori. J Controlled Release 2006;111:1-18.

6. Tadros MI. Controlled-release effervescent floating matrix tablets of ciprofloxacin hydrochloride: development, optimization and in vitro-in vivo evaluation in healthy human volunteers. Eur J Pharm Biopharm 2010;74:332-9.

7. Pawar VK, Kansal S, Garg G, Awasthi R, Singodia D, Kulkarni G. Gastroretentive dosage forms: a review with special emphasis on floating drug delivery systems. Drug Delivery 2011;18:97-110.

8. Manjunath PN, Satish CS, Vasanti S, Preetham AC, Maidu Ras. Formulation and evaluation of simvastatin gastroretentive drug delivery system. Int J Appl Pharm 2017;9:55-60.

9. Albadry AA, Ali WK, Al-Saady FA. Formulation and evaluation of prochlorperazine maleate sustained floating release tablet. Int J Pharm Pharm Sci 2017;9:89-98.

10. Mali AD, Bathe RS. Development and evaluation of gastroretentive floating tablet of a quinapril $\mathrm{HCl}$ by direct compression technique. Int J Pharm Pharm Sci 2017;9:35-46.

11. Wolters K. Drug information handbook with international trade names index. 24thed. Ohio: LEXI-COMP; 2015.

12. Ho HO, Liu CH, Lin HM, Sheu MT. The development of matrix tablets for diclofenac sodium based on an empirical in vitro and in vivo correlation. J Controlled Release 1997;49:149-56.

13. Kapsi SG, Ayres JW. Processing factors in development of solid solution formulation of itraconazole for enhancement of drug dissolution and bioavailability. Int J Pharm 2001;229:193-203.

14. Javadzadeh Y, Jafari-Navimipour B, Nokhodchi A. Liquisolid technique for dissolution rate enhancement of a high dose waterinsoluble drug (carbamazepine). Int J Pharm 2007;341:26-34.

15. Shokri J, Ahmadi P, Rashidi P, Shahsavari M, Rajabi Siahboomi A, Nokhodchi A. Swellable elementary osmotic pump (SEOP): an effective device for delivery of poorly water-soluble drugs. Eur J Pharm Biopharm 2008;68:289-97.

16. Moore F, Okelo G, Colon I, Kushner J. Improving the hardness of dry granulated tablets containing sodium lauryl sulfate. Int J Pharm 2010;400:37-41.

17. Patel VF, Patel NM. Intragastric floating drug delivery system of cefuroxime axetil: in vitro evaluation. AAPS PharmSciTech 2006; 7:118-24.

18. United States Phaemacopeia and National Formulary (USP 39-NF 34). Rockville MD. United States Pharmacopeial Convention; 2015.

19. Bhoi P, Dash RK, Dalai MK. Formulation and in vitro evaluation of oral floating matrix tablets of diclofenac sodium. Int J PharmTech Res 2010;2:2420-8.

20. Hu L, Li L, Yang X, Liu W, Yang J, Jia Y, et al. Floating matrix dosage form for dextromethorphan hydrobromide based on gas forming technique: in vitro and in vivo evaluation in healthy volunteers. Eur J Pharm Sci 2011;42:99-105.

21. Siewert M, Dressman J, Brown CK, Shah VP, FIP, AAPS. FIP/AAPS guidelines to dissolution/in vitro release testing of novel/special dosage forms. AAPS PharmSciTech 2003;4:E7. 
22. Nagarwal RC, Ridhurkar DN, Pandit JK. In vitro release kinetics and bioavailability of gastroretentive cinnarizine hydrochloride tablet. AAPS PharmSciTech 2010;11:294-303.

23. Timmermans J, Andre JM. Factors controlling the buoyancy and gastric retention capabilities of floating matrix capsules: new data for reconsidering the controversy. J Pharm Sci 1994;83:18-24.

24. Li S, Lin S, Daggy BP, Mirchandani HL, Chien TW. Effect of formulation variables on the floating properties of gastric floating drug delivery system. Drug Dev Ind Pharm 2002;28:783-93.

25. Rowe RC, Sheskey PJ, Quinn ME. Handbook of pharmaceutical excipients. 6th ed. Gurnee (IL): Pharmaceutical Press; 2009.

26. Rao GK, Mandapalli PK, Manthri R, Reddy VP. Development and in vivo evaluation of gastroretentive delivery systems for cefuroxime axetil. Saudi Pharm J 2013;21:53-9.

27. Hoffman A, Qadri BA. Gastro-retentive systems. In: James Swarbrick. Ed. Encyclopedia of pharmaceutical technology. 3rd ed. Vol. 1. NY: Informa healthcare; 2007. p. 1850-60.

28. Padmavathy J, Saravanan D, Rajesh D. Formulation and evaluation of ofloxacin floating tablets using HPMC. Int J Pharm Pharm Sci 2011;3:170-3.

29. Peppas NA, Khare AR. Preparation, structure and diffusional behavior of hydrogel in controlled release. Adv Drug Delivery Rev 1993;11:1-35.

30. Bertram U, Bodmeier R. In situ gelling, bioadhesive nasal inserts for extended drug delivery: in vitro characterization of a new nasal dosage form. Eur J Pharm Sci 2006;27:62-71.

31. Hasçiçek C, Yüksel Tilkan G, Türkmen B, Ozdemir N. Effect of formulation parameters on the drug release and floating properties of gastric floating two-layer tablets with acetylsalicylic acid. Acta Pharm 2011;61:303-12.

32. Jagdale SC, Ghorpade SA, Kuchekar BS, Chabukswar AR. Effect of polymer and gas forming agent on floating drug delivery of tramadol hydrochloride using response surface methodology: in vitro and in vivo evaluation. Int J Pharm Appl 2011;2:181-94.

33. Jaimini M, Rana AC, Tanwar YS. Formulation and evaluation of famotidine floating tablets. Curr Drug Delivery 2007;4:51-5.

34. Someshwar K, Chithaluru K, Ramarao T, Kumar K. Formulation and evaluation of effervescent floating tablets of tizanidine hydrochloride. Acta Pharm 2011;61:217-26.

35. Hari V, Sathiyaraj S, Devi R. Lornoxicam gastro-retentive floating matrix tablets: design and in vitro evaluation. J Adv Pharm Technol Res 2011;2:156-62.

36. Mohapatra S, KumarKar R, Mohapatra DK, Sahoo SK, Barik BB. Cefuroxime axetil loaded gastroretentive floating tablets based on hydrophilic polymers: preparation and in vitro evaluation. Braz Arch Biol Technol 2012;55:269-75.

37. Cwiertnia B, Hladon T, Stobiecki M. Stability of diclofenac sodium in the inclusion complex with beta-cyclodextrin in the solid state. J Pharm Pharmacol 1999;51:1213-8.

38. Mura P, Moyano JR, Gonzalez-Rodriguez ML, Rabasco-Alvarez AM, Cirri M, Maestrelli F. Characterization and dissolution properties of ketoprofen in binary and ternary solid dispersions with polyethylene glycol and surfactants. Drug Dev Ind Pharm 2005;31:425-34.

39. Kebebe D, Belete A, Gebre-Mariam T. Evaluation of two olibanum resins as rate controlling matrix forming excipients in oral sustained-release tablets. Ethiop Pharm J 2010;28:95-109. 